\title{
An evaluation of the accelerometer output as a motion artifact signal during photoplethysmograph signal processing control
}

\author{
Muhideen Abbas Hasan ${ }^{1}$, Munther Naif Thiyab ${ }^{2}$, Settar S. Keream ${ }^{3}$ Uzba H Salaman ${ }^{4}$, Kaleid w abid \\ ${ }^{1}$ Basic Sciences, Medical College, Ibn Sina University of Medical and Pharmaceutical sciences, Iraq \\ ${ }^{2,3}$ College of Engineering, University of Anbar, Iraq \\ ${ }^{4} \mathrm{Al}-$ Dour Technical Institute, Northern Technical University, Iraq \\ ${ }^{5}$ Renewable Energy Research Center / University of Anbar, Iraq
}

\begin{tabular}{l}
\hline \hline Article Info \\
\hline Article history: \\
Received Feb 7, 2020 \\
Revised Apr 8, 2020 \\
Accepted Apr 22, 2020 \\
\hline
\end{tabular}

Keywords:

Accelerometer

Adaptive noise cancellation

Covered photo-detector

Noise reflector

PPG signals

\begin{abstract}
Photoplethysmography (PPG) sensors are widely used in medical applications due to their attractive properties such as non-invasiveness, inexpensive, and easy setup. However, they are still inefficient in nonstationary states of important measurements related to cardiovascular assessment. Adaptive noise cancellation (ANC) has existed as a kind of technique to address this issue. Unfortunately, the traditional 3-Axis Accelerometer (ACC) in ANC implementation has failed to provide the real motion artifact (MA) as the main factor for efficient adaptive filtering. In this work, the performance of ACC will be investigated and compared with a new twin photodiodes PPG probe design (TPs-PPD) that has been proven in previous work. The TPs-PPD contained an added covered photodiode (CPD) customized to obtain the MA instead of classic use of ACC. During different motions, PPG data were recorded and processed at the same time by the same two units of adaptive filters using ACC and CPD as noise references. The results indicated a clear failure of the ACC compared to the CPD in determining important features of PPG signal, in addition to the accuracy of signal to noise ratio (SNR) and mean square error (MSE). The CPD was better than ACC as it reduced the MSE by 14 times while the SNR was multiplied 10 times. Without any doubt, it has been proven with evidence that the ACC is not suitable for the processing of human health-related signals while PPG can be used for such purposes.
\end{abstract}

Copyright $@ 2020$ Institute of Advanced Engineering and Science. All rights reserved.

\section{Corresponding Author:}

Muhideen Abbas Hasan,

Department of Basic Sciences,

Ibn Sina University of Medical and Pharmaceutical Sciences,

Baghdad, Iraq.

Email:muhyabass@gmail.com

\section{INTRODUCTION}

Photoplethysmographic (PPG) signal is generated by an optoelectronic device known as a PPG or pulse oximeter sensor used for non-invasive measurement of blood flow in arterial vessels [1]. This device is used to track the resulting cardiovascular wave pulses that would be propagated through the arteries. The pulse oximeter device comprises only a LED light source and photodiode (PD) assembled inside a clip PPG probe. The PD converts around 5\% of the total incident light into an electronic signal which is a combination of the desired PPG signal and noise; therefore, it is classified as a weak signal [2].

PPG signals are utilized to determine the pulse rate, oxygen saturation, respiratory rate (RR), fetal heart rate, etc. [3]. PPG sensors are fallible during movements due to the induced noise caused by motion; this leads to inaccurate readings of the PPG signal and causes false diagnosis [4]. The ANC is one of the several techniques for providing accurate PPG signals $[5,6]$. It depends on active filtering and utilizes the 
adaptive filter. The precondition for the reliable adaptive filter performance is obtaining the originallyinduced noise reference [5,7]. Traditionally, the noise reference signal is provided by an added extra hardware, such 3-axes accelerometer [8,9].

Many attempts have been made towards getting the induced noise signal by synthetic noise from the corrupted PPG signal itself, but it has been found as an inefficient method to meet the sudden changes and relatively high-cost function processing $[10,11]$. The electronic noise effect was noticed while applying an ACC method with such poor signals is not considered; therefore, it does not fully reflect the entire induced noise caused by motion $[12,13]$. PPG signal is rich with important information that can be obtained quickly and inexpensively if properly processed $[14,15]$. Thus, PPG technique still needs further development to be offered as an appropriate alternative to the old ACC approach; its drawbacks ought to be addressed to provide the total induced MA in order to achieve safe and accurate PPG measurements.

In this research, the accelerometer output was evaluated as a motion artifact signal during PPG signal processing in terms of the extent of its validity during PPG signal processing. This was done in comparison with the CPD included within TPs-PPD for the same task. The TPs-PPD comprises two photodiodes; the initial one (IPD) is customized to capture the noisy PPG signal while the other one (CPD) is totally shielded from light effect and customized to obtain only the entire induced MA. The mentioned design has been scientifically justified in terms of time and frequency domains and showed super-sensitivity and recording for likely noise than the ACC $[16,17]$. For practical evaluation, the same data were recorded from healthy volunteers during various activities and processed simultaneously using the CPD and the ACC to get the MA. Twin adaptive filter units were also utilized to achieve true comparison between the results. From the results, CPD was found more capable of identifying the effective noise than the ACC. The CPD minimized the resulting MSE by 14 times while the SNR was increased 10 times in comparison to the ACC for the same processed PPG signal (both outcomes are indicators of the efficiency of the adaptive filter). Hence, the ACC output signal is not suitable for feeding the reference adaptive filter input during PPG signal processing as it requires a high level of accuracy due to its importance in cardiac assessment.

\section{METHODOLOGY}

The methodology of this work will be based on a simple review of the previously designed TPsPPD. Then, the performance of the ACC will be clarified in terms of SNR and MSE compared to the CPD which was included within the mentioned design. This task requires the processing of the same noisy PPG data in the same way and time and utilizing different approaches the ACC and CPD separately to provide the reference noise accompanying the signal to be addressed.

\subsection{The twin photodiodes ppg probe design (TPs-PPD)}

Before proceeding with the evaluation of the ACC, a simple review of the previously designed TPsPPD will be provided for better understanding. In addition to the infra-red- LED (IR-LED), TPs-PPD comprises two similar photodetectors as seen in Figure 1. The first photodetector is the initial (IPD); in all classic PPG probes, it is functionated to detect the PPG signal that is normally accompanied by noise. The second photodetector (CPD) is a component of the TPs-PPD and is properly covered to prevent light penetration effect. According to photodetector characteristic in dark state, the leaking dark current within the CPD was exploited to reflect the entire environmental noise resulting from the motion and the surrounding electromagnetic effects $[18,19]$.

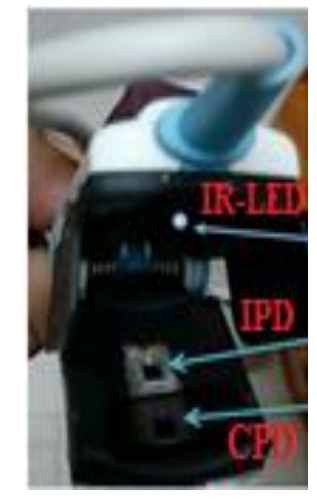

Figure 1. Twin Photodiodes PPG Probe Design (TPs-PPD) 


\subsection{Data acquisition setup}

To carry out the evaluation process for the ACC and CPD, the data acquisition system was first prepared. A hardware and software platform were customized to collect PPG data from 14 healthy volunteers (9 males and 5 females, age 29 \pm 10 years). ACC was tied to the TPDs-PPD that includes the IPD and CPD as illustrated in Figure 2. Noisy PPG signal from IPD and references noise from the ACC and the CPD were simultaneously captured during three types of motions according to the following protocol steps. The data were digitized at $100 \mathrm{~Hz}$ using the DAQ-NI 6341 connected to Lab-View (National Instrument corporation).

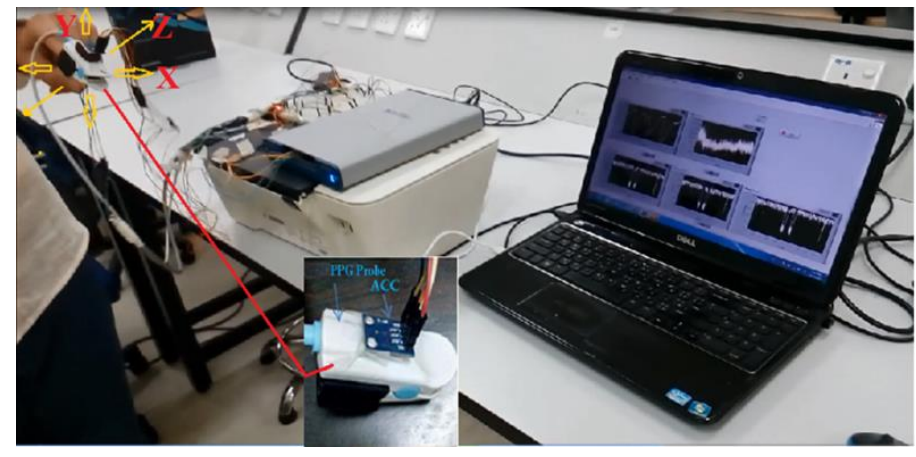

Figure 2. Data acquisition setup

\subsection{Protocol steps}

a) People who have participated in this event were ordered to stand and wear the mentioned PPG sensor by the index finger of the left hand.

b) Participants were asked to move the subject hand at a frequency of $5 \mathrm{~Hz}$ within a space of $20 \mathrm{~cm}$ in both quick and sudden movements; each motion is performed for one minute along the $\mathrm{X}, \mathrm{Y}$ and $\mathrm{Z}$ axes.

c) All captured data were recorded and online processed by two similar units of adaptive filters with a step size of 0.005 and filter length of 256. The first unit to process the raw PPG signal from IPD $V_{\text {IPD }}(\mathrm{n})$ with reference noise was provided by the $C P D V_{C P D}(n)$ while the second unit to process the same raw data $\mathrm{V}_{\mathrm{IPD}}(\mathrm{n})$ with reference noise was provided by the $\operatorname{ACC} \mathrm{V}_{\mathrm{ACC}}(\mathrm{n})$ as revealed in Figure 3.

d) In the current application, the algorithm of least mean squares (LMS) will be adopted as a simpler than Recursive Least Square (RLS) algorithm in adaptive filters applications. LMS algorithm manages the digital filter weight $w[n]$ and adaptively updates the digital filter weights according to (1) [20, 21].

$$
\mathrm{w}[\mathrm{n}+1]=\mathrm{w}[\mathrm{n}]+\mu \mathrm{e}[\mathrm{n}] \mathrm{x}[\mathrm{n}]
$$

where $\mu$ is convergence step size, $x[n]$ is the input vector and $\mathrm{e}[\mathrm{n}]$.

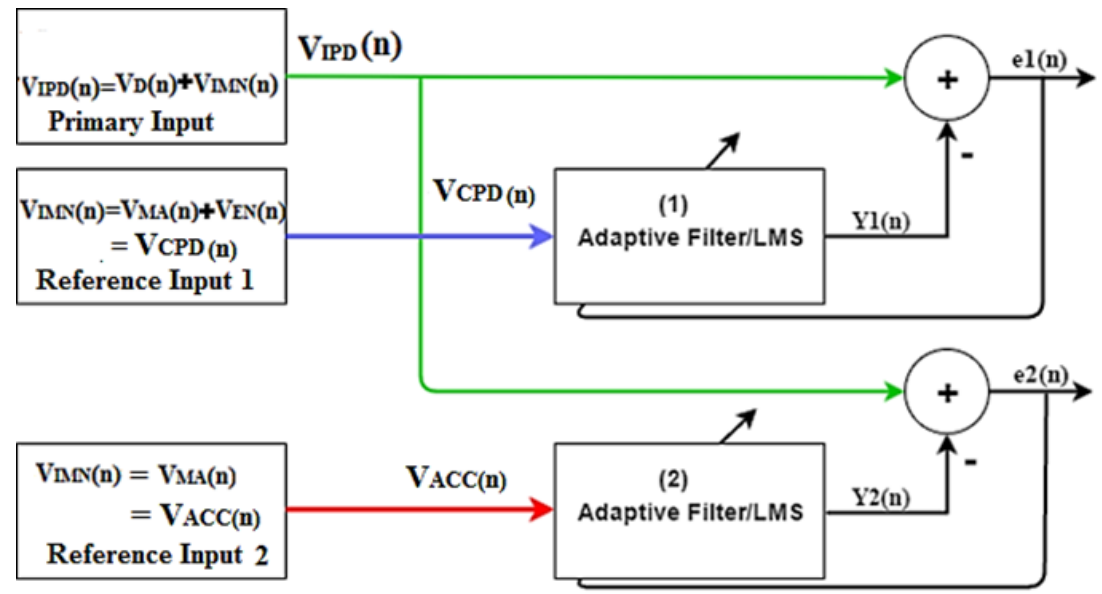

Figure 3. Two similar adaptive filters are designed to process same raw PPG signal $\mathrm{V}_{\mathrm{RPPG}}$ with different reference signals of induced motion noise $\mathrm{V}_{\text {IMN }}$ and motion artifact $\mathrm{V}_{\mathrm{MA}}$

An evaluation of the accelerometer output as a motion artifact signal during ... (Muhideen Abbas Hasan) 


\subsection{The proposed model based on CPD implementation}

The error $\mathrm{e}[\mathrm{n}]$ is the difference between $\mathrm{V}_{\text {IPD }}$ as a primary signal, including the desired signal $\mathrm{V}_{\mathrm{D}}$ and the filtered output $\mathrm{y}(\mathrm{n})$ as the actual reaction signal under the effect of the recent motion artefact reference signals $\operatorname{VCPD}(\mathrm{n})$ and $\operatorname{VACC}(\mathrm{n})$ as shown in (2), (3). The traditional application of MA reference signal in ANC is shown in (4) whereas the resulting model of the presented work is illustrated in (5).

$$
\begin{aligned}
& \mathrm{e}_{1}(\mathrm{n})=\mathrm{V}_{\mathrm{D}}(\mathrm{n})+\mathrm{V}_{C P D}(\mathrm{n})-\mathrm{y}_{1}(\mathrm{n}) \\
& \mathrm{e}_{2}(\mathrm{n})=\mathrm{V}_{\mathrm{D}}(\mathrm{n})+\mathrm{V}_{\mathrm{ACC}}(\mathrm{n})-\mathrm{y}_{2}(\mathrm{n}) \\
& \mathrm{V}_{\mathrm{IMN}}(\mathrm{n})=V_{M A}(n)=V_{A C C}(n)
\end{aligned}
$$

ACC points the induced motion noise $\mathrm{V}_{\mathrm{IMN}}$ only as the motion artefact $V_{M A}$ within $\mathrm{X}, \mathrm{Y}$, and $\mathrm{Z}$ directions.

$$
\mathrm{V}_{\mathrm{IMN}}(\mathrm{n})=\mathrm{V}_{\mathrm{MA}}(\mathrm{n})+V_{E N}(n)
$$

The CPD use can point the induced motion noise $V_{\text {IMN }}$ as the motion artefact $V_{M A}$ and the induced electronic noise $V_{E N}$. where $\mathrm{e}_{1}(\mathrm{n})$ and $\mathrm{e}_{2}(\mathrm{n})$ are the error signals, $\mathrm{V}_{\mathrm{D}}(\mathrm{n})$ is the desired signal, $\mathrm{V}_{\text {IMN }}(\mathrm{n})$ is the likely induced motion noise generated by either the ACC and CPD, $V_{M A}(n)$ is the motion generated by the $\mathrm{ACC}, \mathrm{V}_{\mathrm{EN}}(\mathrm{n})$ is electronic noise [22].

\subsection{Mean square error}

The mean square error (MSE) can be adopted as an indicator of how the filter adapts to a required solution. Small MSE values indicate accurate adaptive filter system modelling, convergence, and prediction of a key solution to the implemented system. Quantization and the measured noise are among the factors that affect the produced minimum MSE. Therefore, MSE is considered a criterion for assessing the performance of the adaptive filter $[23,24]$.

\subsection{Signal-to-noise ratio}

Signal-to-noise ratio (SNR) is the ratio of the measured signal power to the noise power; it is measured to reflect the signal quality index (SQI) of PPG processed signal [25]. Several formulas can be implemented to calculate the signal-to-noise ratio. In this study, the SNR was calculated during the use of ACC and CPD implementation using (6) and (7).

$$
\begin{aligned}
& S N R_{C P D}=20 \log \left(\frac{R M S E_{Y 1}}{R M S E_{e 1}}\right) d B \\
& S N R_{\mathrm{ACC}}=20 \log \left(\frac{\mathrm{RMSE}_{\mathrm{Y} 2}}{\mathrm{RMSE}_{\mathrm{e} 2}}\right) \mathrm{dB}
\end{aligned}
$$

where $\mathrm{SNR}_{\mathrm{CPD}}$, and $\mathrm{SNR}_{\mathrm{ACC}}$ are the signal-to-noise ratio of the first and second adaptive filters, respectively.

$\mathrm{RMSE}_{\mathrm{Y} 1}, \mathrm{RMSE}_{\mathrm{e} 1}, \mathrm{MSE}_{\mathrm{Y} 2}$ and $\mathrm{MSE}_{\mathrm{e} 2}$ are the calculated Root MSE for the filtered and resulted error signals for adaptive filters 1 and 2 , respectively.

\section{RESULTS AND DISCUSSION}

To reveal the ACC drawbacks in reflecting the induced noise usually caused by motion compared to CPD performance, the outcomes of this study will be discussed according to the presented figures in this section.

\subsection{PPG features and signal quality}

Figure 4 showed 10 seconds of processed raw PPG signal (RPPG) pointed in green using ACC and $\mathrm{CPD}$ as noise reflectors during moving of hand along the $\mathrm{Y}$ axis as this state presents the most motion effect. The processed and enhanced PPG signal (En-ACC) using the ACC was presented in red, while the En-CPD, using the CPD, is in blue. The RPPG is already corrupted by motion artefact as it can be noticed to have been deformed as the real peaks of heart beats are missing. Clearly, the signal features in En-CPD showed important details compared to the En-ACC in term of systolic and diastolic peaks which are important for diagnosis as seen in Figure 5.

The criterion for qualified processed signal index is the SNR; whenever it is increased, the signal has been enhanced. Figure 6 clearly showed the improvement of the PPG signal at first iteration (more than 
10 times, $0.8 \mathrm{~dB}$ ) using ACC whereas the value was $8.2(\mathrm{~dB})$ for CPD. Stability was also noted in the second iteration for the CPD method as it recorded $10(\mathrm{~dB})$ while stability for ACC started at $5^{\text {th }}$ iteration and reached $7.5(\mathrm{~dB})$.

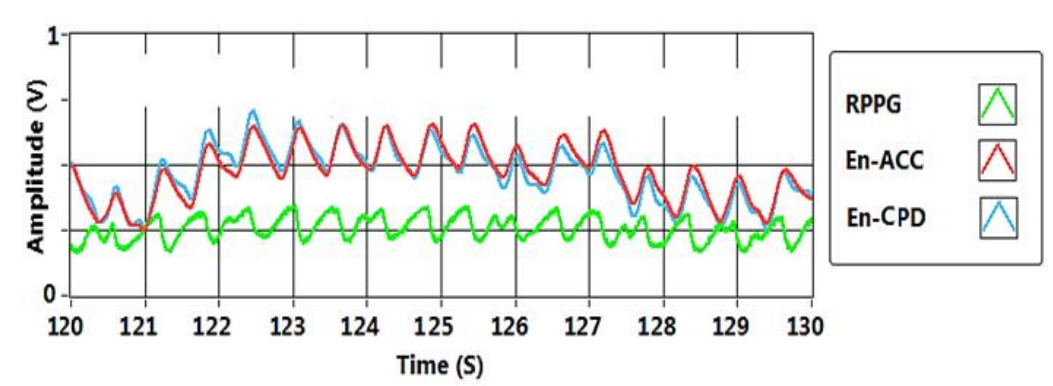

Figure 4. 10 seconds of processed raw PPG signal, using ACC and CPD noise reflectors during moving hand along with $\mathrm{Y}$ axis

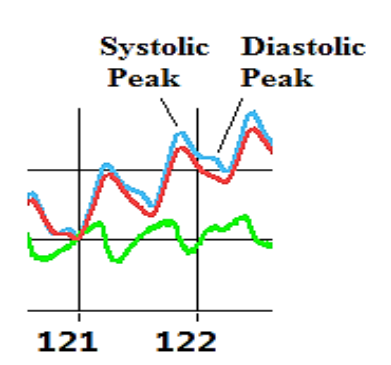

Figure 5. PPG signal features

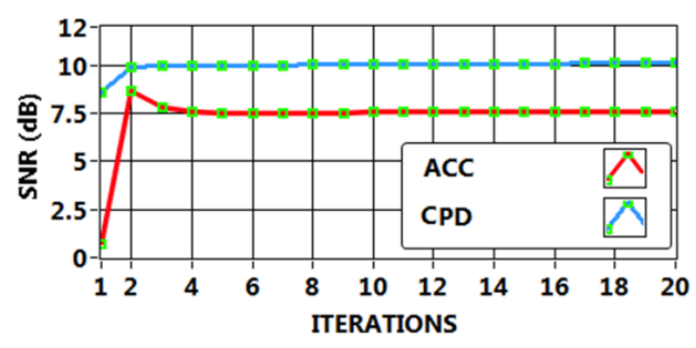

Figure 6. SNR achievements by implementing the two methods (ACC and CPD) as noise reflectors during same raw PPG data processing

\subsection{Mean square error reduction}

Obviously, Figure 7 shows reduction of MSE to $0.4 \mathrm{~V}^{2}$ during the first iteration using the CPD method; this value was 14 times lower than that of ACC. Also, a high stability was achieved during the second iteration with less MSE while ACC method decreased to $0.58 \mathrm{~V}^{2}$ and kept decreasing during 10 iterations without reaching the level dropped by the CPD method. Note that the submitted result is the average score for the 14 people who participated and the average difference was $\pm 0,8$ for SNR and 0.66 for MSE.

Hence, the use of an accelerometer as a reflector for induced noise in critical measurements associated with popular health can never indicate the real induced noise to achieve reliable processing for principal diagnosis. The major function of the accelerometer is to measure the changes in the piezo resistance and hanging mass inside the ACC to point the displacement within three dimensions of $\mathrm{X}, \mathrm{Y}$ and $\mathrm{Z}$ due to movements and gravity effects [26]. The photodetector is an electronic device customized to gauge the changes in photo-resistance due to incident light effect. Both ACC and CPD are electronic devices with different electronic noise according to the Equipartition theorem during any degree of motion [27, 28]. From the discussion so far, in addition to the collected figures, it is evident that the 3 -axis accelerometer cannot be ideal for such critical and sensitive applications.

From the evaluation, ACC is failed to reflect the induced motion noise compared to the CPD method that is more suitable for obtaining the required noise in non-stationary states. The reason behind the achieved priority is the fact that the photodiode and the accelerometer are customized for different functions. Photodiode is produced to detect light and convert it into electronic signal according to the changes in its photo-resistance. In PPG technique, the photodiode follows the variations in blood volume that causes variation in the transmitted light through the subject finger. The ACC is produced to measure the changes in the piezo-resistance and gravity effect to reflect the acceleration within $\mathrm{X}, \mathrm{Y}$ and $\mathrm{Z}$ directions depending on a suspended mass. Accordingly, the expected noise could be identified from the different electronic devices subject to Equipartition Theorem though there may be variations in such weak signals [28]. Therefore, the proposed TPDs-PPD is the proper solution for PPG technique as long as the generated PPG signal and the expected noise are born from similar sources. 


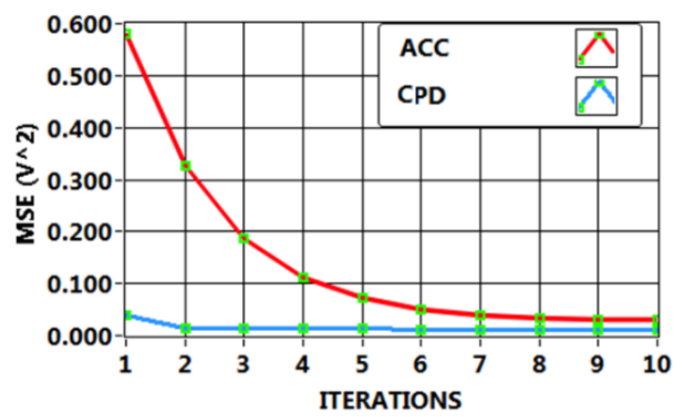

Figure 7. MSE achievements when implementing the two methods (ACC and CPD) as noise reflectors during same raw PPG data processing

\section{CONCLUSION}

This paper presents an evaluation of the accelerometer output as a motion artefact signal during Photoplethysmograph signal processing. Many researchers have adopted the ACC method for the mentioned task, but the results of this study suggest a clear and convincing superiority of the CDD over the ACC method. It can be stated that the use of an accelerometer as a noise reflector is not capable of indicating all the induced noise precisely when processing sensitive PPG signals. The different sources of the corrupted and noise signals lead to difficult in sorting various types of electronic noise. Unreliable measurements result in danger that may arise during the diagnosis as it is related to human health.

Recruiting the same kind of devices can reflect the total induced noise easily as it can achieve a high-level of reading accuracy with less computation; it can also result in economic advantages in terms of low power consumption and low-cost tests. The results showed considerable variations between the two devices in reflecting the noise. Consequently, the ACC is inaccurate in obtaining reassuring results in the processing of such medical signs during the assessment of the health status of individuals.

\section{ACKNOWLEDGEMENTS}

All authors are grateful for the support from Dr. Raja Mohd Taufika Bin Raja Ismail, University Malaysia Pahang, and Eng. Saad Abbas Al-Ani (Iraq) for their valuable efforts in providing the National Instrument hardware and software

\section{REFERENCES}

[1] D. Castaneda, A. Esparza, M. Ghamari, C. Soltanpur, and H. Nazeran, "A review on wearable photoplethysmography sensors and their potential future applications in health care," International journal of biosensors \& bioelectronics, vol. 4, no. 4, pp. 195, 2018.

[2] [M. Elgendi, "On the analysis of fingertip photoplethysmogram signals," Current cardiology reviews, vol. 8, no. 1, pp. 14-25, 2012.

[3] A. W. Setiawan, N. A. Heryanto, F. M. Putra, F. Suyitno, M. A. Martin, Y. S. Raharja, and M. S. Hashuro, "Development of non-occlusive blood pressure monitor with height correction for home used application," Indonesian Journal of Electrical Engineering and Computer Science (IJEECS), vol. 14, no. 2, pp. 921-928, 2019.

[4] B. Manjula, and C. Sharma, "BCG Artifact Removal Using Improved Independent Component Analysis Approach," Indonesian Journal of Electrical Engineering and Computer Science (IJEECS), vol. 5, no. 1, pp. 130-138, 2017.

[5] K. B. Gan, E. Zahedi, and M. A. M. Ali, "Application of Adaptive Noise Cancellation in Transabdominal Fetal Heart Rate Detection Using Photoplethysmography," Adaptive Filtering Applications: IntechOpen, Book, 2011.

[6] S. Fallet, M. Lemay, P. Renevey, C. Leupi, E. Pruvot, and J.-M. Vesin, "An adaptive organization index to characterize atrial fibrillation using wrist-type photoplethysmographic signals." Computing in Cardiology Conference (CinC); IEEE., pp. 337-340, 2016.

[7] N. Khozouie, F. Fotouhi-Ghazvini, and B. Minaei-Bidgoli, “Ontological MobiHealth system," Indonesian J. Elect. Eng. Comput. Sci. (IJEECS), vol. 10, no. 1, pp. 309-319, 2018.

[8] Y. Kong, and K. Chon, "Heart Rate Estimation using PPG signal during Treadmill Exercise." $201941^{\text {st }}$ IEEE Annual International Conference of the Engineering in Medicine and Biology Society (EMBC), pp. 3253-3256, 2019.

[9] D. Jarchi, P. Charlton, M. Pimentel, A. Casson, L. Tarassenko, and D. A. Clifton, "Estimation of respiratory rate from motion contaminated photoplethysmography signals incorporating accelerometry," Healthcare technology letters, vol. 6, no. 1, pp. 19-26, 2019. 
[10] S. Fallet, and J.-M. Vesin, "Adaptive frequency tracking for robust heart rate estimation using wrist-type photoplethysmographic signals during physical exercise." 2015 IEEE Computing in Cardiology Conference (CinC), pp. 925-928, 2015.

[11] R. Yousefi, and M. Nourani, "Methods and systems for extracting venous pulsation and respiratory information from photoplethysmographs," Google Patents, 2019.

[12] Choma, J., "Electronic noise characterization- Part I: System concepts and theory," University of Southern California, Los Angeles, CA, Technical Report\# USC, pp. 02-511, 2011.

[13] J. Song, D. Li, X. Ma, G. Teng, and J. Wei, "PQR signal quality indexes: A method for real-time photoplethysmogram signal quality estimation based on noise interferences," Biomedical Signal Processing and Control, vol. 47, pp. 88-95, 2019.

[14] T. Tamura, Y. Maeda, M. Sekine, and M. Yoshida, "Wearable photoplethysmographic sensors—past and present," Electronics, vol. 3, no. 2, pp. 282-302, 2014.

[15] M. Elgendi, Y. Liang, and R. Ward, "Toward generating more diagnostic features from photoplethysmogram waveforms," Diseases, vol. 6, no. 1, pp. 20, 2018.

[16] M. A. Hasan, F. Samsuri, and K. B. Gan, "A Novel Photoplethysmograph Sensor Probe Design For Motion Artifact Detection: A Comparison Study With Three Axis Accelerometer," Journal of Theoretical and Applied Information Technology, vol. 94, no. 1, pp. 142, 2016.

[17] M. A. Hasan, F. Samsuri, and K. B. Gan, "A Correlation Study on Motion Artifact Using Photodiode and Three Axis Accelerometer Signals," Indian Journal of Science and Technology, vol. 10, no. 7, 2017.

[18] J.-M. Liu, Photonic devices: Cambridge University Press, 2009.

[19] T. Tamir, G. Griffel, and H. L. Bertoni, Guided-wave optoelectronics: device characterization, analysis, and design: Springer Science \& Business Media, 2013.

[20] K. B. Gan, E. Zahedi, and M. A. M. Ali, Application of Adaptive Noise Cancellation in Transabdominal Fetal Heart Rate Detection Using Photoplethysmography: INTECH Open Access Publisher, 2011.

[21] S. A. Vorobyov, A. Cichocki, and Y. V. Bodyanskiy, "Adaptive noise cancellation for multi-sensory signals," Fluctuation and noise letters, vol. 1, no. 1, pp. R13-R24, 2001.

[22] H. Eren, and J. G. Webster, "Measurement, Instrumentation, and Sensors Handbook: Electromagnetic, Optical, Radiation, Chemical, and Biomedical Measurement," 2014.

[23] C. J. Willmott, and K. Matsuura, "Advantages of the mean absolute error (MAE) over the root mean square error (RMSE) in assessing average model performance," Climate research, vol. 30, no. 1 , pp. 79-82, 2005.

[24] W. Harrison, J. Lim, and E. Singer, “A new application of adaptive noise cancellation," IEEE transactions on acoustics, speech, and signal processing, vol. 34, no. 1, pp. 21-27, 1986.

[25] M. Elgendi, "Optimal signal quality index for photoplethysmogram signals," Bioengineering, vol. 3, no. 4, pp. 21, 2016.

[26] M. Andrejašic, "Mems accelerometers." University of Ljubljana Faculty for mathematics and physics, Department of physics, Seminar; 2008.

[27] NA, "Noise of Instrumentation," Imperial College, Autumn, Book, 2008.

[28] P. Barry, and S. Errede, "Measurement of $1 / \mathrm{f}$ noise in carbon composition and thick film resistors," Department of Physics, University of Illinois at Urbana-Champaign, PHYS, vol. 499, 2014. 